PROCEEDINGS OF THE

AMERICAN MATHEMATICAL SOCIETY

Volume 139, Number 9, September 2011, Pages 3317-3326

S 0002-9939(2011)10796-2

Article electronically published on February 11, 2011

\title{
COMMUTING ISOMETRIES OF THE COMPLEX HYPERBOLIC SPACE
}

\author{
WENSHENG CAO AND KRISHNENDU GONGOPADHYAY
}

(Communicated by Michael Wolf)

\begin{abstract}
Let $H_{\mathbb{C}}^{n}$ denote the complex hyperbolic space of dimension $n$. The group $U(n, 1)$ acts as the group of isometries of $H_{\mathbb{C}}^{n}$. In this paper we investigate when two isometries of the complex hyperbolic space commute. Along the way we determine the centralizers.
\end{abstract}

\section{INTRODUCTION}

Let $H_{\mathbb{C}}^{n}$ denote the complex hyperbolic space of dimension $n$. The group $U(n, 1)$ acts as the group of isometries of $H_{\mathbb{C}}^{n}$. In this paper we ask when two isometries of the complex hyperbolic space commute. A related problem is to determine the centralizers. For the two-dimensional complex hyperbolic space, Basmajian-Miner 1. Corollaries 8.2-8.3] determined the conditions for two isometries to commute. In arbitrary dimension, a partial answer to this question was given by Kamiya [5, Proposition 1.11]. However, the answer of Kamiya was far from being complete. In this research paper, we classify the elements which commute with a given isometry and along the way determine the centralizers.

Let $\mathbb{V}$ be a vector space over $\mathbb{C}$ of dimension $n+1$. Let $\mathbb{V}$ be equipped with the standard complex Hermitian form of signature $(n, 1)$. Let $\mathbb{V}_{-}$denote the set of all vectors with negative length under this Hermitian form. The $n$-dimensional complex hyperbolic space $H_{\mathbb{C}}^{n}$ is the image of $\mathbb{V}_{-}$in the projective space $P(\mathbb{V})$. The isometry group $U(n, 1)$ of the Hermitian space $\mathbb{V}$ acts as the isometries of $H_{\mathbb{C}}^{n}$. The actual group of the isometries is $P U(n, 1)=U(n, 1) / Z(U(n, 1))$, where the center $Z(U(n, 1))$ can be identified with the circle group. Thus an isometry $g$ of $H_{\mathbb{C}}^{n}$ lifts to a unitary transformation $\tilde{g}$ in $U(n, 1)$, and in the projective model of $H_{\mathbb{C}}^{n}$ the fixed points of $g$ correspond to eigenvectors of $\tilde{g}$. In the following, we shall often forget the lift and shall use the same symbol for an isometry as well as its lifts. An eigenvalue $\lambda$ of $g \in U(n, 1)$ is called negative if it has an eigenvector in $\mathbb{V}_{-}$.

In the ball model of the hyperbolic space, by Brouwer's fixed point theorem it follows that every isometry $g$ has a fixed point on the closure $\overline{H_{\mathbb{C}}^{n}}$. An isometry $g$ is called elliptic if it has a fixed point on $H_{\mathbb{C}}^{n}$. It is called parabolic, resp. hyperbolic,

Received by the editors January 26, 2010 and, in revised form, January 27, 2010 and August 21, 2010 .

2010 Mathematics Subject Classification. Primary 51M10; Secondary 51F25, 20G20.

Key words and phrases. Complex hyperbolic space, isometries, centralizers.

The first author was supported by the NSF of China (No. 10801107) and the NSF of Guangdong Province (No. 8452902001000043).

(C)2011 American Mathematical Society Reverts to public domain 28 years from publication 
if it is non-elliptic and has exactly one, resp. two, fixed points on the boundary $\partial H_{\mathbb{C}}^{n}$. The conjugacy classification of the isometries of $H_{\mathbb{C}}^{n}$ is known essentially from the work of Chen-Greenberg [2, Theorem 3.4.1]. It follows from the conjugacy classification that the elliptic and hyperbolic elements are semisimple; i.e. their minimal polynomial is a product of linear factors. The parabolic elements are not semisimple.

Let $g$ be elliptic. It follows from the conjugacy classification that all the eigenvalues have norm 1 , and it has a negative eigenvalue. If the negative eigenvalue has multiplicity at least 2 , then it is called a boundary elliptic. Otherwise, $g$ is a regular elliptic; i.e. all the eigenvalues are distinct. Suppose $g$ is hyperbolic. Then it has a complex eigenvalue outside the unit circle and one eigenvalue inside the unit circle. The other eigenvalues lie on the circle; i.e. they have norm one. If all the eigenvalues of $T$ are real, then $g$ is called strictly hyperbolic. Otherwise, it is called a loxodromic. Note that a loxodromic can be written as $g=g_{r} g_{e}$, where $g_{r}$ is strictly hyperbolic and $g_{e}$ is a boundary elliptic. Suppose $g$ is parabolic. If $g$ is unipotent, i.e. all the eigenvalues are 1 , it is called a translation. A translation $g$ is called vertical or non-vertical according as the minimal polynomial is $(x-1)^{2}$ or $(x-1)^{3}$. If $g$ is a non-unipotent parabolic, then it has the Jordan decomposition $g=g_{s} g_{u}$, where $g_{s}$ is semisimple, $g_{u}$ is unipotent in $U(n, 1)$, and $g_{s}$ and $g_{u}$ commute. A non-unipotent parabolic is also called an ellipto-parabolic. A non-vertical translation keeps invariant a unique two-dimensional totally geodesic real subspace, which is called the axis.

A parabolic element which fixes the point ' $\infty$ ' on the boundary is called a Heisenberg translation. Two Heisenberg translations $(\tau, t),(\sigma, s) \in \mathfrak{N}_{n}=\mathbb{C}^{n-1} \times \mathbb{R}$ are called isotropic if $\operatorname{Im}\left(\tau^{*} \sigma\right)=0$. The vertical Heisenberg translations are isotropic with any Heisenberg translation. The isotropy between two non-vertical Heisenberg translations implies that the angle (cf. Goldman [3, p. 36]) between the axes is a non-zero real and that geometrically they are parallel. Suppose $S$ and $T$ are two non-vertical translations of $H_{\mathbb{C}}^{n}$ having the same fixed point. Then they can be simultaneously conjugated to two non-vertical Heisenberg translations. The translations $S$ and $T$ are isotropic if the corresponding Heisenberg translations are isotropic. Our main theorem is the following.

Theorem 1.1. (1) Let $T$ acts as an elliptic isometry of $H_{\mathbb{C}}^{n}$. Then an element $S$ in $U(n, 1)$ commutes with $T$ if and only if it preserves each eigenspace of $T$.

(2) Let $T$ act as a hyperbolic isometry of $H_{\mathbb{C}}^{n}$. Then an element $S$ in $U(n, 1)$ commutes with $T$ if and only if it preserves each eigenspace of $T$, and it acts either as the identity or a strictly hyperbolic on the complex line joining the fixed points of $T$.

(3) Let $T$ be a translation with fixed point $x$. Let $S$ commute with $T$. Then one of the following holds:

(i) $S$ is boundary elliptic such that $S(x)=x$ and $T(f i x(S))=f i x(S)$.

(ii) $S$ is a translation with $S(x)=x$, and $S$ and $T$ are isotropic.

(iii) $S$ is an ellipto-parabolic with fixed point $x$, and it has the Jordan decomposition $S=S_{s} S_{u}$ such that $T\left(f i x\left(S_{s}\right)\right)=f i x\left(S_{s}\right)$ and $T$ and $S_{u}$ are isotropic.

(4) Let $T=T_{s} T_{u}$ be ellipto-parabolic with fixed point $x$. Let $S$ commute with $T$. Then one of the following holds: 
(i) $S$ is boundary elliptic with $S(x)=x, T_{u}(f i x(S))=f i x(S)$ and $T_{s}$ preserves each eigenspace of $S$.

(ii) $S$ is a translation with $S(x)=x, S\left(f i x\left(T_{s}\right)\right)=f i x\left(T_{s}\right)$ and $S$ and $T_{u}$ being isotropic.

(iii) $S$ is an ellipto-parabolic with fixed point $x$, and it has the Jordan decomposition $S=S_{s} S_{u}$ such that $T_{u}\left(f i x\left(S_{s}\right)\right)=f i x\left(S_{s}\right), S_{u}\left(f i x\left(T_{s}\right)\right)$ $=f i x\left(T_{s}\right), T_{u}$ and $S_{u}$ are isotropic, and $T_{s}$ preserves each eigenspace of $S_{s}$.

A useful corollary to the above theorem is the description of the centralizers, up to conjugacy. Following Kulkarni [6], two elements in a group $G$ are said to be in the same $z$-class if their centralizers are conjugate in $G$. The $z$-classes provide useful stratification of the group $G$ and provide better insight about the nature of the action of $G$ on any set $X$; cf. Kulkarni [6]. The $z$-classes of the real hyperbolic space have been classified by Gongopadhyay-Kulkarni [4. The $z$-classes of the isometries of the complex hyperbolic space are obtained from the above theorem.

Corollary 1.2. Let $\mathfrak{N}_{n}$ denote the unipotent subgroup of a stabilizer subgroup of $U(n, 1)$. Let $N$ denote the group of all isotropic translations in $\mathfrak{N}_{n}$. The representatives of the $z$-classes in $U(n, 1)$ are given by:

elliptic: $Z(T)=U\left(r_{1}-1,1\right) \times \Pi_{j=2}^{s} U\left(r_{j}\right), \Sigma_{j=1}^{s} r_{j}=n$.

hyperbolic: $\mathbb{S}^{1} \times \mathbb{R} \times \Pi_{j=1}^{s} U\left(r_{j}\right), \Sigma_{j=1}^{s} r_{j}=n-1$.

vertical translation: $Z(T)=U(n-1) \ltimes \mathfrak{N}_{n}$.

non-vertical translation: $Z(T)=\left[\mathbb{S}^{1} \times U(n-2)\right] \ltimes N$.

The $z$-class of a non-unipotent element is obtained as $Z(T)=Z\left(T_{s}\right) \cap Z\left(T_{u}\right)$, where $T_{s}, T_{u}$ are the semisimple and the unipotent parts of $T$ in its Jordan decomposition.

In Section 2, we review the models of $H_{\mathbb{C}}^{n}$ and the Heisenberg similarity transformations. In Section 3, several properties of the Heisenberg similarities are given. Especially, we show that a unipotent isometry and an elliptic isometry commute if and only if they mutually fix their fixed point sets. This is crucial to the proof of our main theorem. In Section 4, we prove our main theorem, and Corollary 1.2 is established in Section 4.3 .

\section{Preliminaries}

In this section we give the necessary background material on complex hyperbolic space. More extensive facts may be found in [2, 3, 7,.

\subsection{The complex hyperbolic space.}

2.1.1. The ball model. Let $\mathbb{V}$ be a vector space of dimension $(n+1)$ over $\mathbb{C}$ equipped with the complex Hermitian form of signature $(1, n)$,

$$
\langle z, w\rangle=-\bar{z}_{0} w_{0}+\bar{z}_{1} w_{1}+\cdots+\bar{z}_{n} w_{n}
$$

where $z$ and $w$ are the column vectors in $\mathbb{V}$ with entries $z_{1}, \ldots, z_{n+1}$ and $w_{1}, \ldots$, $w_{n+1}$, respectively. Define

$$
\mathbb{V}_{0}=\{z \in \mathbb{V} \mid\langle z, z\rangle=0\}, \mathbb{V}_{+}=\{z \in \mathbb{V} \mid\langle z, z\rangle>0\}, \mathbb{V}_{-}=\{z \in \mathbb{V} \mid\langle z, z\rangle<0\}
$$

Let $\mathbb{P}(\mathbb{V})$ be the projective space obtained from $\mathbb{V}$, i.e., $\mathbb{P}(\mathbb{V})=\mathbb{V}-\{0\} / \sim$, where $u \sim v$ if there exists $\lambda$ in $\mathbb{C}^{*}$ such that $u=v \lambda$, and $\mathbb{P}(\mathbb{V})$ is equipped with the 
quotient topology. Let $\pi: \mathbb{V}-\{0\} \rightarrow \mathbb{P}(\mathbb{V})$ denote the projection map. We define $H_{\mathbb{C}}^{n}=\pi\left(\mathbb{V}_{-}\right)$. The boundary $\partial H_{\mathbb{C}}^{n}$ in $\mathbb{P}(\mathbb{V})$ is $\pi\left(\mathbb{V}_{0}\right)$.

If $z=\left(z_{0}, \ldots, z_{n}\right) \in \mathbb{V}_{-}$, the condition $-\left|z_{0}\right|^{2}+\sum_{k=1}^{n}\left|z_{i}\right|^{2}<0$ implies $z_{0} \neq 0$. Therefore we may define a set of coordinates $\zeta=\left(\zeta_{1}, \ldots, \zeta_{n}\right)$ on $H_{\mathbb{C}}^{n}$ by $\zeta_{i}(\pi(z))=$ $z_{i} z_{0}^{-1}$. This identifies $H_{\mathbb{C}}^{n}$ with the ball

$$
\mathbb{B}_{\mathbb{C}}^{n}=\left\{\zeta=\left.\left(\zeta_{1}, \ldots, \zeta_{n}\right)\left|\sum_{k=1}^{n}\right| \zeta_{i}\right|^{2}<1\right\}
$$

With this identification the map $\pi: \mathbb{V}_{-} \rightarrow H_{\mathbb{C}}^{n}$ has the coordinate representation $\pi(z)=\zeta$, where $\zeta_{i}=z_{i} z_{0}^{-1}$. The boundary $\partial H_{\mathbb{C}}^{n}$ is identified with

$$
\mathbb{S}_{\mathbb{C}}^{n-1}=\left\{\zeta=\left(\zeta_{1}, \ldots, \zeta_{n}\right)\left|\sum_{k=1}^{n}\right| \zeta_{i}^{2} \mid=1\right\}
$$

Let $\left\{f_{1}, \ldots, f_{n}\right\}$ denote the standard basis of $\mathbb{C}^{n}$. Under the projection map, $f_{1}=$ $(1,0, \ldots, 0)$ and $-f_{1}=(-1,0, \ldots, 0)$ correspond to boundary points of $\partial H_{\mathbb{C}}^{n}$.

2.1.2. The Siegel domain model. This model for $H_{\mathbb{C}}^{n}$ is obtained in the same way as above except that the Hermitian form on $\mathbb{V}$ under consideration is

$$
\langle z, w\rangle_{2}=w^{*} J z=\bar{w}_{0} z_{n}+\bar{w}_{1} z_{1}+\cdots+\bar{w}_{n-1} z_{n-1}+\bar{w}_{n} z_{0},
$$

where $J$ is the Hermitian matrix

$$
J=\left(\begin{array}{ccc}
0 & 0 & 1 \\
0 & I_{n-1} & 0 \\
1 & 0 & 0
\end{array}\right) .
$$

The two models are related by a Cayley transformation; cf. [2, 3, 7]. The two Hermitian forms are referred to as first and second Hermitian forms respectively. When there is no confusion, we shall forget the subscript from the Hermitian forms, and it should be clear from the context.

A unitary transformation $g$ is an automorphism of $\mathbb{C}^{n, 1}$, that is, a linear bijection such that $\langle g(z), g(w)\rangle=\langle z, w\rangle$ for all $z$ and $w$ in $\mathbb{C}^{n, 1}$. We denote the group of all unitary transformations by $U(n, 1)$.

The isometry group of $H_{\mathbb{C}}^{n}$ is $P U(n, 1)=U(n, 1) / Z(U(n, 1))$. However, since the elements of $U(n, 1)$ are linear, it is convenient to deal with $U(n, 1)$ rather than $P U(n, 1)$.

Suppose $g \in U(n, 1)$. In the Siegel domain model, for all $z$ and $w$ in $\mathbb{V}$.

$$
w^{*} J z=\langle z, w\rangle=\langle g z, g w\rangle=w^{*} g^{*} J g z,
$$

where $*$ denotes the Hermitian transpose. Letting $z$ and $w$ vary over a basis of $\mathbb{V}$, we see that $J=g^{*} J g$. From this we find $g^{-1}=J^{-1} g^{*} J$. That is,

$$
g^{-1}=\left(\begin{array}{ccc}
\bar{d} & \beta^{*} & \bar{b} \\
\delta & A^{*} & \gamma \\
\bar{c} & \alpha^{*} & \bar{a}
\end{array}\right) \text { for } g=\left(\begin{array}{ccc}
a & \gamma^{*} & b \\
\alpha & A & \beta \\
c & \delta^{*} & d
\end{array}\right) \in U(n, 1),
$$

where $A$ is an $(n-1) \times(n-1)$ matrix, $\alpha, \beta, \gamma, \delta$ are column vectors in $\mathbb{C}^{n-1}$, and $a, b, c, d \in \mathbb{C}$. 
2.2. The Heisenberg similarity transformations. The $(2 n-1)$-dimensional Heisenberg group $\mathfrak{N}_{n}$ is $\mathbb{C}^{n-1} \times \mathbb{R}$ with the group law

$$
\left(\zeta_{1}, v_{1}\right)\left(\zeta_{2}, v_{2}\right)=\left(\zeta_{1}+\zeta_{2}, v_{1}+v_{2}+2 \operatorname{Im}\left(\zeta_{2}^{*} \zeta_{1}\right)\right)
$$

The boundary of $H_{\mathbb{C}}^{n}$ may be identified with the one-point compactification of the Heisenberg group. We define horospherical coordinates on the complex hyperbolic space as follows:

$$
\begin{gathered}
\psi: \mathfrak{N}_{n} \times \mathbb{R}_{+} \rightarrow \mathbb{V}_{0} \cup \mathbb{V}_{-}, \\
\psi(\zeta, v, u)=\left(\begin{array}{c}
\left(-|\zeta|^{2}-u+i v\right) / 2 \\
\zeta \\
1
\end{array}\right), \psi(\infty)=\left(\begin{array}{c}
1 \\
0 \\
\vdots \\
0
\end{array}\right) .
\end{gathered}
$$

We also define the origin $o$ to be the point in $\partial H_{\mathbb{C}}^{n}$ with horospherical coordinates $(0,0,0)$, that is,

$$
\psi(o)=(0, \ldots, 0,1)^{t} .
$$

In this way the $\partial H_{\mathbb{C}}^{n}$ is identical with $\mathfrak{N}_{n} \times\{0\} \cup \infty$.

The unitary group $U(n-1)$ acts on horospherical coordinates as Heisenberg rotation, whose action is given by

$$
R_{U}:(\zeta, v, u) \rightarrow(U \zeta, v, u) .
$$

The corresponding matrix in $U(n, 1)$ acting on $\mathbb{C}^{n, 1}$ is

$$
R_{U}=\operatorname{diag}(1, U, 1) \text {. }
$$

The fixed point set of such a map is a totally geodesic subspace of $H_{\mathbb{C}}^{n}$ passing through $o$ and $\infty$.

The positive real numbers $r \in \mathbb{R}^{+}$act as Heisenberg dilation $D_{r}$ on horospherical coordinates, whose action is given by

$$
D_{r}:(\zeta, v, u) \rightarrow\left(r \zeta, r^{2} v, r^{2} u\right) .
$$

As a matrix $D_{r}$ is given by

$$
D_{r}=\operatorname{diag}\left(r, I_{n-1}, \frac{1}{r}\right) .
$$

The $(\tau, t) \in \mathfrak{N}_{n}$ acts as a Heisenberg translation on horospherical coordinates, whose action is given by

$$
T_{(\tau, t)}:(\zeta, v, u) \rightarrow((\tau, t)(\zeta, v), u)=\left(\tau+\zeta, v+k+2 \operatorname{Im}\left(\zeta^{*} \tau\right), u\right) .
$$

As a matrix $T_{(\tau, t)}$ is given by

$$
\left(\begin{array}{ccc}
1 & -\tau^{*} & \frac{-|\tau|^{2}+t i}{2} \\
0 & I_{n-1} & \tau \\
0 & 0 & 1
\end{array}\right) .
$$

When $\tau=0, T_{(\tau, t)}$ is a vertical Heisenberg translation. Otherwise, it is a nonvertical Heisenberg translation. It is easy to see that the subgroup $\mathfrak{T}$ formed by vertical Heisenberg translations is the center of $\mathfrak{N}_{n}$ and $\mathfrak{T} \approx \mathbb{R}$.

A parabolic element is called screw parabolic if it is the product of a Heisenberg translation and a Heisenberg rotation. When $n \geq 3$, we will show that a screw parabolic which is the product of a non-vertical Heisenberg translation and a 
Heisenberg rotation cannot be conjugated to a screw parabolic which is the product of a vertical Heisenberg translation and Heisenberg rotation in Section 3 .

Let $U(n, 1)_{\infty}$ denote the isotropy group of $U(n, 1)$ at $\infty$. Then

$$
U(n, 1)_{\infty}=\left[\mathbb{S}^{1} \times \mathbb{R}^{+} \times U(n-1)\right] \ltimes \mathfrak{N}_{n},
$$

where $\ltimes$ denotes the semidirect product of groups. Thus every element $g$ in $U(n, 1)_{\infty}$ can be uniquely written as $g=D_{r} R_{U} T_{(\tau, t)}$, where $R_{U}$ is elliptic, $D_{r}$ is hyperbolic and $T_{(\tau, t)}$ is unipotent in $U(n, 1)_{\infty}$. Elements in $U(n, 1)_{\infty}$ are called the Heisenberg similarity transformations. If $D_{r}$ is the identity in the above decomposition, $g$ is called a Heisenberg isometry. It is easy to see that elements $g$ fixing $o$ and $\infty$ are of the form

$$
g=\operatorname{diag}(\mu, A, \lambda),
$$

where $\bar{\mu} \lambda=1$ and $A \in U(n-1)$.

\section{Commuting elements of translations}

Throughout this section, we use the second Hermitian form of the hyperbolic space.

Lemma 3.1. Let $T, S \in P U(n, 1)$. If $S$ commutes with $T$, then

$$
T(f i x(S))=f i x(S), S(f i x(T))=f i x(T) .
$$

Proof. Let $x \in f i x(S)$. Then $S T(x)=T S(x)=T(x)$, which implies that $T(f i x(S))$ $\subset$ fix $(S)$. Also we have $T^{-1}(x)=T^{-1} S(x)=S T^{-1}(x)$, which implies that $T^{-1}(f i x(S)) \subset f i x(S)$. Thus $T(f i x(S))=f i x(S)$.

By matrix computation, we have the following observation:

$$
T_{(\tau, t)} R_{U}=R_{U} T_{\left(U^{*} \tau, t\right)} .
$$

Using this we have the following.

Lemma 3.2. Let $T=R_{U} T_{(\tau, t)}$ and $S=R_{V} T_{(\sigma, s)}$. Then $S$ commutes with $T$ if and only if

$$
V U=U V,\left(V^{*} \tau, t\right)(\sigma, s)=\left(U^{*} \sigma, s\right)(\tau, t) .
$$

Lemma 3.3. Let $T=R_{U} T_{(\tau, t)}$ and $T_{(\tau, t)} \neq I$. If $U \tau=\tau$, then $T$ is parabolic.

Proof. Suppose that $U \tau=\tau$ and $T$ is elliptic. Then $T$ is boundary elliptic, and by its action on the horospherical coordinate there exists a point $(\zeta, v, u)$ in $H_{\mathbb{C}}^{n}$ such that

$$
U(\tau+\zeta)=\zeta, t+2 \operatorname{Im}\left(\zeta^{*} \tau\right)=0 .
$$

Hence we have that

$$
\zeta^{*} \tau=\zeta^{*} U^{*} U \tau=\left(\zeta^{*}-\tau^{*}\right) \tau=\zeta^{*} \tau-|\tau|^{2} .
$$

This implies that $\tau=0$ and $t=0$. This contradiction implies $T$ is parabolic.

Remark 3.4. Note that $T=R_{U} T_{(\tau, t)}$ with $U \tau=\tau$ and $R_{U} \neq I_{n-1}$ can also be conjugated to the form $R_{U} T_{\left(\tau_{1}, t_{1}\right)}$ such that $U \tau_{1} \neq \tau_{1}$. We give the reason as follows. Let $\mathbb{V}_{1}(U)=\left\{x \in \mathbb{C}^{n-1} \mid U x=x\right\}$. For $\tau \neq 0, U \tau=\tau$, by linear algebraic theory the matrix equation

$$
(U-I) x=\tau
$$


has no solution $x$. This is $\sigma+\tau-U \sigma \neq 0$. When $n \geq 3$, there exists a non-zero $\sigma \notin \mathbb{V}_{1}(U)$. For such $\sigma, T_{(\sigma, s)} T T_{(\sigma, s)}^{-1}$ is

$$
R_{U} T_{\left(U^{*} \sigma+\tau-\sigma, t-2 \operatorname{Im}\left(\sigma^{*} U \sigma\right)+4 \operatorname{Im}\left(4 \tau^{*} \sigma\right)\right)}=T_{\left(\sigma+\tau-U \sigma, t-2 \operatorname{Im}\left(\sigma^{*} U^{*} \sigma\right)+4 \operatorname{Im}\left(4 \tau^{*} \sigma\right)\right)} R_{U},
$$

where $\tau_{1}=U^{*} \sigma+\tau-\sigma \neq(\sigma+\tau-U \sigma)=U \tau_{1}$. We also have

$$
\begin{gathered}
R_{V} T R_{V}^{-1}=R_{V U V^{*}} T_{(V \tau, t)}=T_{(V \tau, t)} R_{V U V^{*}}, \\
D_{r} T D_{r}^{-1}=R_{U} T_{\left(r \tau, r^{2} t\right)}=T_{\left(r \tau, r^{2} t\right)} R_{U} .
\end{gathered}
$$

The above two conjugations cannot make the unipotent part of $T=R_{U} T_{(\tau, t)}$ to be a vertical Heisenberg translation. The above observations imply that, when $n \geq 3$, we cannot conjugate a screw parabolic $T=R_{U} T_{(\tau, t)}$ with $U \tau=\tau \neq 0$ to the form of $R_{U^{\prime}} T_{\left(0, t^{\prime}\right)}$. By the three conjugations (3.2), (3.3), (3.4), we know that the definition of isotropic in Section 1 is well defined. We also mention that a boundary elliptic element $R_{v} T_{(\sigma, s)}$ can be conjugated in $U(n, 1)_{\infty}$ to diagonal form.

By the above, it also follows that, for $n \geq 3$, there are two types of screw parabolic elements $T=R_{U} T_{(\tau, t)}$ with $U \tau=\tau, \tau \neq 0$ and $\tau=0$, respectively.

In what follows, we are concerned about which element can commute with a unipotent parabolic element $T$. By conjugation, we may assume that $T$ fixes $\infty$; that is, $T=R_{U} T_{(\tau, t)}$. By Lemma 3.1, if $S$ commutes with $T$, then we must have

$$
T(f i x(S))=f i x(S), S(\infty)=\infty .
$$

If $S$ is hyperbolic, then by $T(f i x(S))=f i x(S)$, we get a contradiction. This implies that $S$ is Heisenberg isometry which can be written uniquely as $S=R_{V} T_{(\sigma, s)}$.

Lemma 3.5. Let $T$ and $S$ be unipotent. Then $S T=T S$ if and only if $S$ and $T$ have the same fixed point and they are isotropic.

Proof. Suppose $T S=S T$. By Lemma 3.1, they have a common fixed point $x$. Let $K \in U(n, 1)$ such that $K(x)=\infty$. Then $K T K^{-1}$ and $K S K^{-1}$ must be of the forms $T_{(\tau, t)}$ and $T_{(\sigma, s)}$. It follows from Lemma 3.2 that $S$ and $T$ are isotropic. The converse is obvious from the definition of isotropic.

Lemma 3.6. Let $T$ be unipotent and $S$ elliptic. Then $S T=T S$ if and only if $T(f i x(S))=f i x(S)$ and $S(f i x(T))=f i x(T)$.

Proof. By Lemma 3.1, we only need to prove the necessity. Without loss of generality, let $T=T_{(\tau, t)}$ and $S=R_{V} T_{(\sigma, s)}$ with $V \sigma \neq \sigma$. The action of $S$ on the horospherical coordinate $(\zeta, v, u)$ is

$$
S:(\zeta, v, u) \rightarrow\left(V \sigma+V \zeta, v+s+2 \operatorname{Im}\left(\zeta^{*} \sigma\right), u\right) .
$$

Let $(\zeta, v, u) \in H_{\mathbb{C}}^{n}$ be a fixed point of $S$; then

$$
V(\sigma+\zeta)=\zeta, s+2 \operatorname{Im}\left(\zeta^{*} \sigma\right)=0 .
$$

If $T(f i x(S))=f i x(S)$, then $T((\zeta, v, u))=\left(\tau+\zeta, t+v+2 \operatorname{Im}\left(\zeta^{*} \tau\right), u\right) \in f i x(S)$. That is,

$$
V(\sigma+\tau+\zeta)=\tau+\zeta, s+2 \operatorname{Im}\left((\tau+\zeta)^{*} \sigma\right)=0 .
$$

This implies that $V \tau=\tau, \operatorname{Im}\left(\tau^{*} \sigma\right)=0$. By Lemma 3.2, this implies that $S T=$ $T S$. 


\section{Proof of the MAIN THEOREM}

In this section, we shall prove our main theorem case by case. Mainly using Lemma 3.2 and the proof of our main theorem, we shall determine the centralizers up to conjugacy.

4.1. The semisimple cases. To classify the commuting elements of the semisimple isometries, we use the first Hermitian form $\langle$,$\rangle . It is customary to call a subspace$ $\mathbb{W}$ (i) time-like if the restriction $\left.\langle\rangle\right|_{,\mathbb{W}}$ is non-degenerate and indefinite, (ii) spacelike if $\left.\langle\rangle\right|_{,\mathbb{W}}>0$, (iii) light-like if $\left.\langle\rangle\right|_{,\mathbb{W}}=0$. In particular, a vector $v \in \mathbb{V}$ is time-like, space-like or light-like according as $\langle v, v\rangle\langle 0,\langle v, v\rangle>0$ or $\langle v, v\rangle=0$.

Suppose $T$ is semisimple, i.e. elliptic or hyperbolic. Suppose $S$ commutes with $T$. Let $\lambda$ be an eigenvalue of $T$ and let $\mathbb{V}_{\lambda}$ be the eigenspace to $\lambda$. Let $v \in \mathbb{V}_{\lambda}$. Then $T S(v)=S T(v)=S(\lambda v)=\lambda S(v)$, i.e. $S(v) \in \mathbb{V}_{\lambda}$. Thus each eigenspace of $T$ is $S$-invariant, and each eigenspace of $S$ is $T$-invariant.

Let $\lambda$ and $\mu$ be two distinct eigenvalues of $T$. For $v \in \mathbb{V}_{\lambda}, w \in \mathbb{V}_{\mu}$, note that

$$
\langle v, w\rangle=\langle T v, T w\rangle=\bar{\lambda} \mu\langle v, w\rangle .
$$

Thus if $\bar{\lambda} \mu \neq 1$, then $\mathbb{V}_{\lambda}$ and $\mathbb{V}_{\mu}$ are orthogonal to each other.

(1) Let $T$ be elliptic. Suppose each eigenspace of $T$ is $S$-invariant. We claim that $S$ commutes with $T$. Suppose the distinct eigenvalues of $T$ are $e^{i \theta_{j}}, j=1, \ldots, s$, where $e^{i \theta_{1}}$ is assumed to be time-like and the rest are space-like. For each $j$, let $\mathbb{V}_{j}$ be the eigenspace to $e^{i \theta_{j}}$. Then $\mathbb{V}$ has the orthogonal decomposition: $\mathbb{V}=\bigoplus_{j=1}^{s} \mathbb{V}_{j}$. Let $Q_{j}$ denote the Hermitian form $\left.\langle\rangle\right|_{,\mathbb{V}_{j}}$. Since $S$ is an isometry of $\mathbb{V}$, each $\mathbb{V}_{j}$ is $S$-invariant, and hence $S$ preserves the orthogonal decomposition and also the restricted metric on each of the summands. Thus $S$ commutes with $T$ if and only if $\left.S\right|_{\mathbb{V}_{j}}$ commutes with $\left.T\right|_{\mathbb{V}_{j}}$. Let $Q_{j}$ denote the restriction of $Q$ to $\mathbb{V}_{j}$. Since $\left.T\right|_{\mathbb{V}_{j}}=e^{i \theta_{j}} I_{\mathbb{V}_{j}}$, it is a central element in $U\left(\mathbb{V}_{j}, Q_{j}\right)$. Hence any $\left.S\right|_{\mathbb{V}_{j}}$ in $U\left(\mathbb{V}_{j}, Q_{j}\right)$ commutes with $\left.T\right|_{\mathbb{V}_{j}}$. This establishes the claim.

(2) Suppose $T$ is hyperbolic. Let $L_{r}$, resp. $L_{r^{-1}}$, denote the one-dimensional eigenspaces corresponding to the light-like eigenvalues $r$, resp. $r^{-1}$. Let $\mathbb{V}_{r}=$ $L_{r}+L_{r^{-1}}$. Let $\mathbb{V}_{j}$ denote the eigenspace of $T$ corresponding to the space-like eigenvalue $e^{i \theta_{j}}$. In this case $\mathbb{V}$ has the orthogonal decomposition

$$
\mathbb{V}=\mathbb{V}_{r} \oplus \bigoplus_{j=1}^{s} \mathbb{V}_{j},
$$

where $\mathbb{V}_{r}=L_{r}+L_{r^{-1}}$. Clearly $S$ keeps $\mathbb{V}_{r}$ invariant. For $j=r, 1, \ldots, s$, let $Q_{j}$ denote the Hermitian form $\left.\langle\rangle\right|_{,\mathbb{V}_{j}}$. As above, we see that $\left.S\right|_{\mathbb{V}_{j}}$ commutes with $\left.T\right|_{\mathbb{V}_{j}}$ for each $j=1, \ldots, s$. Hence $S$ commutes with $T$ if and only if $\left.S\right|_{\mathbb{V}_{r}}$ commutes with $\left.T\right|_{\mathbb{V}_{r}}$. Since $S$ keeps the eigenspaces of $T$ invariant, it keeps the light-like lines $L_{r}$ and $L_{r^{-1}}$ invariant. Hence $\left.S\right|_{\mathbb{V}_{r}}$ commutes with $\left.T\right|_{\mathbb{V}_{r}}$ if and only if it is either a strictly hyperbolic or a central element in $U\left(\mathbb{V}_{r}, Q_{r}\right)$. Consequently the assertion follows.

4.2. Parabolic case. We shall use the Siegel domain model, i.e. the second Hermitian form, to deal with the parabolic case. Let $T$ be a parabolic isometry. Let $S$ commute with $T$. Suppose $S$ is semisimple. It cannot be hyperbolic, for otherwise $T$ would have two fixed points on the boundary. So $S$ must be elliptic. We assert that $S$ cannot be a regular elliptic, for otherwise $T$ fixes the unique fixed point of $S$, which is impossible. Hence $S$ must be a boundary elliptic. 
By conjugation, we assume its unique fixed point to be $\infty$, i.e. $T$ is a Heisenberg isometry. If $T$ is ellipto-parabolic, it has the unique Jordan decomposition $T=$ $T_{s} T_{u}=T_{u} T_{s}$, where $T_{s}$ and $T_{u}$ are its semisimple and unipotent components, respectively. From the Jordan decomposition it follows that an element commutes with $T$ if and only if it commutes with both $T_{s}$ and $T_{u}$. Now the assertions follow from Lemma 3.1 Lemma 3.5 and Lemma 3.6

This completes the proof of the main theorem.

\subsection{The centralizers: Proof of Corollary 1.2.}

4.3.1. Elliptic elements. Let $T$ be elliptic. Then we have seen that $\mathbb{V}$ has an orthogonal decomposition $\mathbb{V}=\bigoplus_{j=1}^{s} \mathbb{V}_{j}$, where for each $j, \mathbb{V}_{j}$ is the eigenspace to $e^{i \theta_{j}}$, and let $\theta_{1}$ be the negative eigenvalue. Let $\operatorname{dim} \mathbb{V}_{j}=r_{j}$. For each $j,\left.T\right|_{\mathbb{V}_{j}}$ is a central element in $U\left(\mathbb{V}_{j}, Q_{j}\right)$, which we identify with $U\left(r_{1}-1,1\right)$, resp. $U\left(r_{j}\right)$, for $j=1$, resp. $>1$. Hence

$$
Z(T)=\Pi_{j=1}^{s} Z\left(\left.T\right|_{\mathbb{V}_{j}}\right)=U\left(r_{1}-1,1\right) \times \Pi_{j=2}^{s} U\left(r_{j}\right) .
$$

4.3.2. Hyperbolic elements. Let $T$ be hyperbolic. Then $\mathbb{V}$ has the orthogonal decomposition $\mathbb{V}=\mathbb{V}_{r} \oplus \bigoplus_{j=1}^{s} \mathbb{V}_{j}$, where $V_{r}$ is the 2-dimensional subspace spanned by the light-like eigenvectors and $\mathbb{V}_{j}$ are the eigenspaces spanned by space-like eigenvectors to eigenvalues $e^{i \theta_{j}}, j=1, \ldots, s$. Hence $Z(T)=Z\left(\left.T\right|_{\mathbb{V}_{r}}\right) \times \Pi Z\left(\left.T\right|_{\mathbb{V}_{j}}\right)$. Let $\operatorname{dim} \mathbb{V}_{j}=r_{j}$. As above, we identify it with $U\left(r_{j}\right)$. Note that $\left.T\right|_{\mathbb{V}_{r}}$ acts as a hyperbolic element in $U\left(\mathbb{V}_{r}, Q_{r}\right) \equiv U(1,1)$; hence $Z\left(\left.T\right|_{\mathbb{V}_{r}}\right)=\mathbb{S}^{1} \times \mathbb{R}$. Therefore

$$
Z(T)=\mathbb{S}^{1} \times \mathbb{R} \times \Pi_{j=1}^{s} U\left(r_{j}\right) .
$$

4.3.3. Parabolic elements. First note that the centralizer of $U(n, 1)$ is

$$
\mathbb{S}^{1}=\{\mu I|| \mu \mid=1\} .
$$

(1) Suppose $T$ is a vertical translation. Since $T$ can be conjugated to vertical Heisenberg translation, by Lemma 3.2. up to conjugation in $P U(n, 1)$,

$$
Z(T)=U(n-1) \ltimes \mathfrak{N}_{n} .
$$

Alternatively, using the linear group $U(n, 1)_{\infty}$, we choose the following subgroups:

$$
\begin{gathered}
\hat{\mathbb{S}}^{1}=\left\{g \in U(n, 1)\left|g=\operatorname{diag}\left(\mu, I_{n-1}, \mu\right),\right| \mu \mid=1\right\}, \\
\hat{U}(n-1)=\{g \in U(n, 1) \mid g=\operatorname{diag}(1, A, 1), A \in U(n-1)\} .
\end{gathered}
$$

By direct computation, following the approach of Chen-Greenberg [2, section 4.2], one can see that every element $S$ in $Z(T)$ can be written uniquely as $S=A B P$, $A \in \hat{\mathbb{S}}^{1}, B \in \hat{U}(n-1), P \in \mathfrak{N}_{n}$.

Thus we have, up to conjugation in $U(n, 1)$,

$$
Z(T)=\left[\hat{\mathbb{S}}^{1} \times \hat{U}(n-1)\right] \ltimes \mathfrak{N}_{n} .
$$

(2) Suppose $T$ is a non-vertical translation. Let $T=T_{(\tau, t)}$ and $S \in Z(T)$. Let

$$
N=\left\{T_{\sigma, s} \in \mathfrak{N}_{n} \mid \tau^{*} \sigma \in \mathbb{R}\right\} .
$$

Note that $\mathfrak{T}$ is a subgroup of $N$. It follows from Section 3 that, up to conjugation, $S=R_{V} T_{(\sigma, s)}$ with $\tau \neq 0, V \tau=\tau$, and $\tau^{*} \sigma \in \mathbb{R}$. Using linear algebraic methods, 
there exists a unitary matrix $U$ such that $U \tau=(|\tau|, 0, \ldots, 0)^{t}$; that is, $U V U^{*} e_{1}=e_{1}$. Thus

$$
E_{\tau}=\{V \in U(n-1) \mid V \tau=\tau\} \approx U(n-2),
$$

which implies that, up to conjugation in $P U(n, 1)$,

$$
Z(T)=U(n-2) \ltimes N .
$$

Alternatively, in the linear group $U(n, 1)_{\infty}$, let

$$
\hat{E}_{a}=\left\{g \in U(n, 1)_{\infty} \mid g=\operatorname{diag}(\mu, A, \mu), A a=\mu a\right\} .
$$

By direct computation, one can see that every element $S$ in $Z(T)$ can be written uniquely as $S=B P$, where $B \in \hat{E}_{a}, P \in N$. Further we see that $\hat{E}_{a}$ can be identified with the group $\mathbb{S}^{1} \times U(n-2)$. Hence, up to conjugation in $U(n, 1)$,

$$
Z(T)=\left[\mathbb{S}^{1} \times U(n-2)\right] \ltimes N .
$$

4.3.4. A topological description of the centralizer of $T_{\sigma, s}$. Let $\langle,\rangle_{o}$ denote the Euclidean norm in $\mathbb{C}^{n-1}$, i.e. $\langle x, y\rangle_{o}=x^{*} y$. Thus we can identify

$$
N / \mathfrak{T}=\left\{c \in \mathbb{C}^{n-1} \mid\langle a, c\rangle_{o} \in \mathbb{R}\right\} .
$$

We identify $\mathbb{C}^{n-1}=\mathbb{R}^{2(n-1)}$ and let $\langle,\rangle_{o}$ be the usual Euclidean norm on $\mathbb{R}^{2(n-1)}$. Then $\langle a, c\rangle_{o}=r$ is a $(2 n-3)$-dimensional affine hyperspace orthogonal to $a$, and hence $N / \mathfrak{T} \approx \mathbb{R} \times \mathbb{R}^{2(n-2)} \approx \mathbb{R} \times \mathbb{C}^{n-2}$. Thus

$$
N \approx\left[\mathbb{R} \times \mathbb{C}^{n-2}\right] \ltimes \mathfrak{T} \approx\left[\mathbb{R} \times \mathbb{C}^{n-2}\right] \ltimes \mathbb{R} .
$$

Hence

$$
Z(T) \approx\left[\mathbb{S}^{1} \times U(n-2)\right] \ltimes\left[\left(\mathbb{R} \times \mathbb{C}^{n-2}\right) \ltimes \mathbb{R}\right] .
$$

\section{REFERENCES}

[1] A. Basmajian and R. Miner, Discrete subgroup of complex hyperbolic motions. Invent. Math., 131(1) (1998), 85-136. MR.1489895 (99e:32039)

[2] S. S. Chen and L. Greenberg, Hyperbolic spaces. Contributions to analysis, Academic Press, New York, 1974, 49-87. MR0377765 (51:13934)

[3] W. M. Goldman, Complex hyperbolic geometry. Oxford University Press, 1999. MR 1695450 (2000g:32029)

[4] K. Gongopadhyay and R. S. Kulkarni, z-Classes of isometries of the hyperbolic space. Conform. Geom. Dyn. 13 (2009), 91-109. MR.2491719 (2010e:51028)

[5] S. Kamiya, Notes on elements of $U(1, n ; C)$. Hiroshima Math. J. 21 (1991), 23-45. MR.1091431 (93e:22020b)

[6] R. S. Kulkarni, Dynamical types and conjugacy classes of centralizers in groups. J. Ramanujan Math. Soc. 22 (1) (2007), 35-56. MR2312547 (2008e:20001)

[7] J. R. Parker, On the volumes of cusped, complex hyperbolic manifolds and orbifolds. Duke Math. J. 94 (3) (1998), 433-464. MR1639519 (99i:32030)

School of Mathematics and Computational Science, Wuyi University, Jiangmen, Guangdong 529020, People's Republic of China

E-mail address: wenscao@yahoo.com.cn

Indian Institute of Science Education and Research (IISER) Mohali, Transit Campus: MGSiPAP Complex, Sector-26, Chandigarh 160019, India

E-mail address: krishnendu@iisermohali.ac.in, krishnendug@gmail.com 